OPEN ACCESS

Edited by:

Eleonora Napoli,

University of California, Davis,

United States

Reviewed by:

Boyang Wu

Washington State University,

United States

Elizabeth Ellen Billett,

Nottingham Trent University,

United Kingdom

*Correspondence:

Yue Hou

houyue@mail.neu.edu.cn

Bing Wang

wangbing@mail.neu.edu.cn

${ }^{\dagger}$ These authors have contributed equally to this work

Specialty section:

This article was submitted to

Cellular Biochemistry,

a section of the journal

Frontiers in Molecular Biosciences

Received: 17 December 2019

Accepted: 25 March 2020

Published: 30 April 2020

Citation:

Jia C, Li G, Jiang R, Liu X, Yuan $Q$, Le W, Hou Y and Wang $B$ (2020) B-Cell Receptor-Associated

Protein 31 Negatively Regulates the Expression of Monoamine

Oxidase A Via R1.

Front. Mol. Biosci. 7:64.

doi: 10.3389/fmolb.2020.00064

\section{B-Cell Receptor-Associated Protein 31 Negatively Regulates the Expression of Monoamine Oxidase A Via R1}

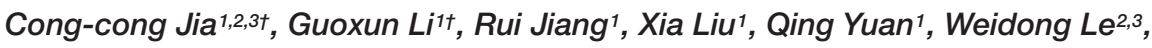 \\ Yue Hou ${ }^{1,4 *}$ and Bing Wang ${ }^{1 *}$ \\ ${ }^{1}$ College of Life and Health Sciences, Northeastern University, Shenyang, China, ${ }^{2}$ Center for Clinical Research on \\ Neurological Diseases, The First Affiliated Hospital, Dalian Medical University, Dalian, China, ${ }^{3}$ Liaoning Provincial Key \\ Laboratory for Research on the Pathogenic Mechanisms of Neurological Diseases, The First Affiliated Hospital, Dalian \\ Medical University, Dalian, China, ${ }^{4}$ Key Laboratory of Data Analytics and Optimization for Smart Industry, Ministry \\ of Education, Northeastern University, Shenyang, China
}

B-cell receptor-associated protein 31 (Bap31) is a three trans-membrane protein of the endoplasmic reticulum (ER). Patients who have loss of function of Bap31 suffered from X-linked syndrome, such as motor and intellectual disabilities, dystonia, and sensorineural deafness. However, the underlying mechanism of Bap31 on X-linked syndrome remains unclear. Here, we found that a total of 21 proteins ( 9 up-regulated and 12 down-regulated proteins) related with X-linked syndrome were screened from shRNA-Bap31 transfected cells with the isobaric tags for relative and absolute quantification (iTRAQ) technique. One gene with the greatest change trend, monoamine oxidase A (MAOA), was identified. MAOA expression was up-regulated by Bap31 knockdown. However, Bap31 did not affect the ubiquitination degradation of MAOA protein. Of note, Bap31 selectively regulated the expression of cell division cycle associated 7-like (R1/RAM2/CDCA7L/JPO2, a transcriptional repressor of MAOA) and the binding activity of R1 with MAOA promoter, thereby affecting MAOA expression. This study demonstrates the molecular mechanisms of Bap31 in MAOA via R1 and supports the potential function of Bap31 on X-linked syndrome.

\section{Keywords: Bap31, MAOA, iTRAQ, R1, X-linked syndrome}

\section{INTRODUCTION}

B-cell receptor-associated protein 31 (Bap31) gene is located from 153,700,492 to $153,724,746 \mathrm{bp}$ on the long (q) arm of the X chromosome in humans (Adachi et al., 1996). The protein is a 28$\mathrm{kDa}$ polytopic integral protein of the endoplasmic reticulum (ER), comprised of a hydrophobic $\mathrm{N}$-terminus and an alpha-helical C-terminus contained with a KKXX motif (Adachi et al., 1996; Maatta et al., 2000). Bap31 is evolutionarily conserved and is known as Yet3p in yeast (Wilson and Barlowe, 2010). It transports certain membrane proteins from the ER to other cellular components, such as cellubrevin (Annaert et al., 1997), CD11b/CD18 (Zen et al., 2004), 
MHC-I (Abe et al., 2009) and protein tyrosine phosphataselike B (Wang et al., 2004). A previous study showed that the caspase cleavage product of Bap31 (p20) induces the fission and apoptosis of mitochondria (Breckenridge et al., 2003). The study of conditional knockout mice of Bap31 indicates that Bap31 has functions in T-cell activation via T-cell receptor signaling (Niu et al., 2017). Bap31, as an important member of the ER-associated degradation (ERAD) (Wakana et al., 2008), promotes the mutant of cystic fibrosis transmembrane regulator (CFTR $\Delta$ F508) to degradation via the Derlin1 complex (Wang et al., 2008). Our former study demonstrates that Bap31 regulates one of the central nervous system disease-related gene (valosin-containing protein, VCP) expression (Jia et al., 2018). Moreover, the contiguous deletion of ABCD1 DXS1357E (Bap31) leads to X-linked syndromes (Corzo et al., 2002; Iwasa et al., 2013). Mutations in Bap31 induce a severe X-linked phenotype, such as dystonia, deafness, and central hypomyelination (Cacciagli et al., 2013).

Monoamine oxidase A (MAOA) is an outer mitochondria membrane protein. Its mutation causes X-linked familial exudative vitreoretinopathy and Norrie disease (Chen et al., 1993, 1995). MAOA is a flavoenzyme that catalyzes the oxidative deamination of biogenic amines, such as serotonin, dopamine, and norepinephrine (Son et al., 2008). The substrates of MAOA are important factors in neural signal transmission; people with an abnormal expression of MAOA exhibit phenotypes, including autism (Verma et al., 2014), an aggressive behavior (Zhang et al., 2017) or depression (Gupta et al., 2016). The variable number of tandem repeats in the promoter region of MAOA frequently affects the expression of MAOA and induces the abnormal behaviors of males (Schluter et al., 2016; Manca et al., 2018). Moreover, cell division cycle associated 7-like (R1/RAM2/CDCA7L/JPO2) competitively binds the binding sites of trans-acting transcription factor 1 (SP1) with the human MAOA promoter, resulting in the down-regulation of the MAOA expression level (Ou et al., 2006a). Ou et al. (2006b) found that MAOA and its transcriptional repressor R1 participate in apoptotic signaling pathways. Furthermore, MAOA activates the Shh-IL6-Rankl signaling pathway in the promotion of prostate cancer metastasis (Wu et al., 2017).

The isobaric tags for relative and absolute quantification (iTRAQ) technique, one of the new proteomics approaches, has been widely used in the analysis of whole differential proteins among the different groups (Wiese et al., 2007). In the present study, iTRAQ was used to detect and analyze the whole differential proteins between shRNA-Bap31 transfected cells and control cells. In order to study the function of Bap31 on X-linked diseases, we screened the genes associated with X-linked diseases from the iTRAQ analyzed results. There were 21 proteins associated with X-linked diseases (9 up-regulated and 12 down-regulated proteins) regulated by Bap31. MAOA is one of them and up-regulated by Bap31 knockdown. Bap31 regulated the expression of MAOA via affecting the binding activity of R1 with the MAOA promoter. In conclusion, our results may demonstrate the mechanism of Bap31 on MAOA-associated $\mathrm{X}$-linked diseases.

\section{MATERIALS AND METHODS}

\section{Cell Culture}

N2a, HEK-293T, and SH-SY5Y cells were maintained in Dulbecco's modified Eagle's medium (DMEM, Gibco, MA, United States) with $10 \%$ fetal bovine serum (Hyclone, Life Technologies, CA, United States) and 1\% pen-strep solution (Biological Industries, CT, United States) at $37^{\circ} \mathrm{C}$ in $5 \% \mathrm{CO}_{2}$. The $\mathrm{N} 2$ a cells that stably knock down Bap31 were cultured in DMEM medium with $10 \mu \mathrm{M}$ puromycin (Thermo, MA, United States) under normal conditions. The SH-SY5Y cells stably expressing Bap31-Flag and the HEK293T cells stably expressing MAOAHA were selected with $100 \mu \mathrm{g} / \mathrm{ml}$ G418/geneticin (Thermo) in DMEM medium under normal conditions.

\section{Plasmid Construction and Transfection}

The fragments of R1 or MAOA-HA coding sequences were generated by polymerase chain reaction (PCR) and then ligated to pcDNA3.1(-) vector. The MAOA promoter fragment was amplified from HEK293T cells DNA and ligated to pGL3basic plasmid. The shRNA fragments targeted to Bap31 or R1 were designed and subcloned into pLKO.1-puro plasmid. All plasmids were confirmed by sequencing before use (Genewiz Biotechnology Co., Ltd., Suzhou, China). Overexpression plasmids and/or shRNA vectors $(2 \mu \mathrm{g})$ targeted to the specific genes were transfected by Lipofectamine 6000, provided by Beyotime Biotechnology (Shanghai, China), according to the manufacturer's instructions. The cells transfected with control vectors were used as control groups.

\section{Dual Luciferase Reporter Assay}

HEK-293T cells stably expressing MAOA-HA transfected with shRNA or overexpression plasmids $(2 \mu \mathrm{g})$ targeted to Bap31 for $48 \mathrm{~h}$. Then, the MAOA promoter fragment luciferase reporter plasmid $(0.5 \mu \mathrm{g})$ and the phRL-SV40 vector (the transfection efficiency control, $0.05 \mu \mathrm{g}$ ) were co-transfected to the abovementioned cells with Lipofectamine 6000 for $48 \mathrm{~h}$. The dual-luciferase assay kit (Beyotime) was used to detect the luciferase activities with a plate reader (BioTek, VT, United States). Three experiments were repeated and each group was set for three repeats. The firefly luciferase signal was normalized to the Renilla luciferase signal.

\section{Real-Time PCR}

Total RNA was extracted from the different samples by using TRIzol reagent (Ambion, MA, United States). Two micrograms was synthesized to cDNA with the GoScript ${ }^{\mathrm{TM}}$ Reverse Transcription System (Promega, WI, United States) according to the manufacturer's instructions. The mRNA levels of the genes were analyzed by the GoTaq $^{\circledR}$ qPCR Master Mix (Promega) with a CFX96 Touch ${ }^{\text {TM }}$ Real-time PCR Detection System (BioRad Laboratories, CA, United States). Three experiments were repeated and each group was set for three repeats. The results were analyzed by the $2^{-\Delta \Delta \mathrm{Ct}}$ formula. The primers of the genes used in this study were shown in Table 1 (Genewiz Biotechnology Co., Ltd.). 
TABLE 1 | Primers used in this study.

\begin{tabular}{|c|c|c|}
\hline Name & Forward primers $\left(5^{\prime}-3^{\prime}\right)$ & Reverse primers $\left(5^{\prime}-3^{\prime}\right)$ \\
\hline Real-time PCR primers of MAOA (mouse) & cagtggagtggctacatgga & acatccttggactcaggctc \\
\hline Real-time PCR primers of MAOA (human) & aattcagcggcttccaatgg & tttccgggcaagaatgaagc \\
\hline Real-time PCR primers of Bap31 (mouse, human) & tccacatgaagcttttccgt & aaatgagagtcaccaggcgt \\
\hline Real-time PCR primers of GAPDH (mouse) & agtgtttcctcgtcccgtag & gccgtgagtggagtcatact \\
\hline Real-time PCR primers of GAPDH (human) & tcgtggaaggactcatgacc & atgatgttctggagagcccc \\
\hline MAOA-HA plasmids primers & ggctagcatggagaatcaagagaaggc & $\begin{array}{l}\text { ggaattctcaagcgtaatctggaacatcgtatgggtacata- } \\
\text { gaccgtggcaggagct }\end{array}$ \\
\hline R1 plasmids primers & ggctagcatggagttggcgactcgct & cgggatccttaattgtcttctaccagctcc \\
\hline shRNA-R1 plasmids primers & $\begin{array}{l}\text { ccggcagacgacgtcaccgtatatcttcgaagatatacggt- } \\
\text { gacgtcgtctgtttttg }\end{array}$ & $\begin{array}{l}\text { aattcaaaaacagacgacgtcaccgtatatcttcgaagatat- } \\
\text { acggtgacgtcgtctg }\end{array}$ \\
\hline MAOA luciferase plasmids primers & ggctagcagctgccgacacaaggacatt & gaagatctcccttctatcaactcccccg \\
\hline shRNA-Bap31 (human) plasmids primers & $\begin{array}{l}\text { ccggatcgatgccgtgcgcgaaattct } \\
\text { cgagaatttcgcgcacggcatcgattttttg }\end{array}$ & $\begin{array}{l}\text { aattcaaaaaatcgatgccgtgcgcgaaattc } \\
\text { tcgagaatttcgcgcacggcatcga }\end{array}$ \\
\hline R1 element & agctgccgacacaaggacat & cccttctatcaactcccc \\
\hline
\end{tabular}

\section{Western Blot}

Cells were lysed in RIPA buffer [1\% TritonX-100, $50 \mathrm{mM}$ Tris (pH 7.4), $150 \mathrm{mM} \mathrm{NaCl}, 1 \mathrm{mM} \mathrm{EDTA}$, and $0.1 \% \mathrm{SDS}$ ] containing protease inhibitor cocktails (Thermo) and incubated on ice for $30 \mathrm{~min}$. After centrifugation at $12,000 \mathrm{rpm}$ for $15 \mathrm{~min}$ at $4^{\circ} \mathrm{C}$, part of the supernatant was used to detect the protein concentration by an Enhanced BCA Protein Assay Kit (Beyotime) with a plate reader (BioTek, VT, United States) at $562 \mathrm{~nm} .5 \times$ SDS buffer was added to the remaining supernatant and then boiled for $10 \mathrm{~min}$. Equal amounts of the total protein $(30-50 \mu \mathrm{g})$ lysates were then separated by $8-10 \%$ SDS-PAGE and transferred onto $0.45 \mu \mathrm{M}$ polyvinylidine fluoride membranes (Millipore, MA, United States). Then, the membranes were blocked with 5\% skim milk for $1 \mathrm{~h}$ at room temperature, washed three times with Trisbuffered saline containing Tween-20 (TBST) buffer, incubated with primary antibodies at $4^{\circ} \mathrm{C}$ overnight, washed three times with TBST buffer, and incubated with the secondary antibodies for $1 \mathrm{~h}$ at room temperature. After that, the bands were visualized with a ChemiDoc ${ }^{\mathrm{TM}}$ XRS + system (Bio-Rad Laboratories, CA, United States) using an ECL detection kit (Tanon, Shanghai, China). The intensities of the bands targeted to the proteins were calculated and normalized to that of $\beta$-actin using Image Lab software (Bio-Rad). Three experiments were repeated. The antibodies used in this study were as follows: rabbit anti-MAOA (Abcam, MA, United States), rabbit anti-R1 (Abcam), rabbit anti-Sp1 (Wanlei Biotechnology Co., Ltd., Shenyang, China), mouse anti-ub (Santa Cruz Biotechnology, TX, United States), mouse anti- $\beta$-actin (Abcam), chicken anti-Bap31, mouse antiHA (Abcam), and the secondary antibodies (Thermo).

\section{Immunoprecipitation}

HEK-293T cells overexpressing MAOA-HA were transfected with shRNA or overexpression plasmids $(2 \mu \mathrm{g})$ targeted to Bap31 for $48 \mathrm{~h}$. The whole-cell lysates were lysed in an immunoprecipitation lysis buffer (Beyotime Biotechnology) containing a protease inhibitor and centrifuged at $4^{\circ} \mathrm{C}$ for $15 \mathrm{~min}$ at $12,000 \mathrm{rpm}$. The supernatant was incubated with mouse antiHA (Abcam) antibody overnight at $4{ }^{\circ} \mathrm{C}$ and then incubated with the protein $\mathrm{A} / \mathrm{G}$ Sepharose (Beyotime) for $4^{\circ} \mathrm{C}$ overnight. The immunoprecipitated proteins were washed three times with lysed buffer, and equal amounts of total protein $(50 \mu \mathrm{g})$ lysates were eluted with $1 \times$ SDS loading buffer and boiled for $10 \mathrm{~min}$. Western blot was used to analyze the samples.

\section{Flow Cytometry}

The cells were resuspended in $200 \mu \mathrm{l}$ of $2 \%$ paraformaldehyde (PFA) in phosphate buffer saline (PBS), incubated on ice for $15 \mathrm{~min}$, and then stained with anti-MAOA diluted in PBS with $1 \%$ saponin on ice water bath at $4^{\circ} \mathrm{C}$ for $1 \mathrm{~h}$, followed by the fluorescein-isothiocyanate (FITC)-conjugated secondary antibody on ice water bath at $4^{\circ} \mathrm{C}$ for $45 \mathrm{~min}$. The solutions were washed three times with PBS containing 2\% bovine serum albumin (BSA), resuspended in PBS, and then detected with a flow cytometry (Becton Dickinson, NJ, United States).

\section{Protein Digestion, TMT Labeling, and LC-MS/MS Analysis}

All samples were homogenized in a lysis buffer $[8 \mathrm{M}$ urea, $1 \%$ Triton-100, $10 \mathrm{mM}$ dithiothreitol (DTT), and $1 \%$ protease inhibitor]. After centrifugation at $20,000 \times g$ for $10 \mathrm{~min}$ at $4^{\circ} \mathrm{C}$, the supernatant was precipitated with cold 15\% TCA for $2 \mathrm{~h}$ at $-20^{\circ} \mathrm{C}$, followed by centrifugation at $4^{\circ} \mathrm{C}$ for $10 \mathrm{~min}$. The precipitate was washed three times with cold acetone and then re-dissolved in buffer [ $8 \mathrm{M}$ urea and $100 \mathrm{mM}$ triethylammonium bicarbonate (TEAB), $\mathrm{pH} 8.0]$, and the protein concentration was determined with the BCA protein assay reagent (Beyotime).

For digestion, the protein solution was reduced with $10 \mathrm{mM}$ DTT (Sigma, NY, United States) at $37^{\circ} \mathrm{C}$ for $1 \mathrm{~h}$ and alkylated with $20 \mathrm{mM}$ iodoacetamide (Sigma) in darkness at room temperature for $45 \mathrm{~min}$. Then, the protein sample was diluted by adding $100 \mathrm{mM}$ TEAB (Applied Biosystems, Milan, Italy) to a urea (Sigma) concentration of less than $2 \mathrm{M}$. Finally, trypsin (Promega) was added at a ratio of 1:50 to the proteins for the first digestion overnight, followed by a second 4-hour digestion with a 1:100 trypsin-to-protein mass ratio. Then, $100 \mu \mathrm{g}$ protein for each sample was digested with trypsin for the following 
experiments. The peptide was desalted by Strata X C18 SPE column (Phenomenex, CA, United States), vacuum-dried, and reconstituted in $0.5 \mathrm{M} \mathrm{TEAB}$ and labeled according to the manufacturer's protocol for 6-plex TMT kit (Thermo). The protein samples were labeled as 130 (control-1), 129 (control-2), 131 (control-3), 131 (shRNA-Bap31-1), 128 (shRNA-Bap31-2), and 130 (shRNA-Bap31-3).

The sample was then fractionated into 80 fractions by high-pH reverse-phase HPLC using Agilent 300Extend C18 $4.6 \times 100 \mathrm{~mm}$ column ( $5 \mu \mathrm{m}$, Agilent, CA, United States) with a gradient of 2 to $60 \%$ acetonitrile in $10 \mathrm{mM}$ ammonium bicarbonate, $\mathrm{pH} \mathrm{10,} \mathrm{over}$ $80 \mathrm{~min}$. Then, the peptides were combined into 18 fractions and dried by vacuum centrifuging.

The peptides were dissolved in $0.1 \%$ FA and loaded onto a reversed-phase pre-column (Acclaim PepMap 100, Thermo Scientific). Peptide separation was performed using a reversedphase analytical column (Acclaim PepMap RSLC, Thermo Scientific) at a constant flow rate of $280 \mathrm{nl} / \mathrm{min}$ on an EASY-nLC 1000 ultra-performance liquid chromatography (UPLC) system. The gradient was comprised of an increase from 7 to $20 \%$ solvent $\mathrm{B}(0.1 \% \mathrm{FA}$ in $98 \% \mathrm{ACN})$ over $24 \mathrm{~min}, 20$ to $35 \%$ in $8 \mathrm{~min}, 35$ to $80 \%$ in $5 \mathrm{~min}$, and holding at $80 \%$ for $3 \mathrm{~min}$. The resulting peptides were analyzed with a $\mathrm{Q}$ Exactive ${ }^{\mathrm{TM}}$ hybrid quadrupoleOrbitrap mass spectrometer (ThermoFisher Scientific).

The peptides were analyzed by tandem mass spectrometry (MS/MS) in Q Exactive ${ }^{\mathrm{TM}}$ (Thermo) coupled to the UPLC. The intact peptides were detected in the Orbitrap at a resolution of 70,000 . The peptides were selected for MS/MS using NCE settings as 27,30 , and 33 . The resolution of ion fragments detected in the Orbitrap was 17,500. A data-dependent procedure that alternated between one MS scan followed by $20 \mathrm{MS} / \mathrm{MS}$ scans was applied for the top 20 precursor ions above a threshold ion count of $1.0 \mathrm{E} 4$ in the MS survey scan with $30.0 \mathrm{~s}$ dynamic exclusion. The electrospray voltage applied was $2.0 \mathrm{kV}$. Automatic gain control was used to prevent overfilling of the ion trap, and 5E4 ions were accumulated for generation of MS/MS spectra. For MS scans, the $\mathrm{m} / \mathrm{z}$ scan range was 350 to 1,800 . The fixed first mass was set as $100 \mathrm{~m} / \mathrm{z}$.

\section{Chromatin Immunoprecipitation}

HEK-293T cells were co-transfected with shRNA-Bap31 and R1 over-expression plasmids or Bap31 overexpression plasmids and shRNA-R1 plasmids for $48 \mathrm{~h}$. ChIP assay was performed according to the manufacturer's protocol (Beyotime). The ChIP primer (Table 1) sets were checked for linear amplification and designed to amplify the region of the human MAOA promoter.

\section{Database Search and Bioinformatics Analysis}

Proteins with a fold change of $1.5(p<0.05)$ between the two groups were identified as differentially expressed. The resulting MS/MS data were processed using Mascot search engine (v.2.3.0). The results of the tandem mass spectra were searched against the Uniprot mouse database (16,717 sequences). The differentially regulated proteins were classified by Gene Ontology (GO) annotation derived from the UniProt-GOA database into three categories: biological process, cellular compartment, and molecular function. The subcellular and gene localization of differentially regulated proteins was analyzed.

\section{Immunofluorescence}

For the immunofluorescence stain, the cells were fixed with $2 \%$ PFA in PBS buffer for $30 \mathrm{~min}$, washed with PBS three times, and permeated with $0.2 \%$ Triton $\mathrm{X}-100$ for $30 \mathrm{~min}$ at room temperature. After that, the cells were washed with PBS three times, blocked with $2 \%$ BSA in PBS buffer and then stained with an anti-R1 antibody followed by the FITC-conjugated secondary antibody (Abcam). The nuclei were stained with Hoechst 33342 (Sigma-Aldrich). The fluorescence images were taken under a confocal laser scanning microscope (Leica, IL, United States). The fluorescence intensity was analyzed with the ImageJ software $(\mathrm{NIH})$.

\section{Statistical Analysis}

Independent-sample Student's tests and one-way ANOVA were used to analyze the significance of the results. All experimental data were reported as the mean \pm standard error of the mean (SEM) (significance: ${ }^{*} p<0.05,{ }^{* *} p<0.01$ ).

\section{RESULTS}

\section{The Proteins Involved in X-Linked Diseases Regulated by Bap31}

Bap31 is widely expressed in various cells, such as B cells (Adachi et al., 1996), T cells (Niu et al., 2017), and neurons (Manley and Lennon, 2001). To explore the unknown function of Bap31, we used the Bap31 knockdown plasmid (pL/shRNAmouse-shRNA-Bap31) and the antibiotics to construct Bap31 knockdown cells (shRNA-Bap31). Real-time PCR and Western blot were used to analyze the knockdown efficiency of Bap31; the mRNA and protein levels of Bap31 were significantly decreased by 79 and $80 \%$ compared with that of the control cells, respectively (Figures 1A,B). These results demonstrate that the shRNA-Bap31 transfected cells are qualified as Bap31 effectively knockdown cells.

Isobaric tags for relative and absolute quantification is a recently developed technique in the quantitative proteomics which measures fold changes in protein expression among different groups (Moulder et al., 2018). In this study, iTRAQ was used to detect the differentially expressed proteins between shRNA-Bap31 transfected cells and control cells. In total, 4,349 proteins were identified and 2,989 proteins were quantified. When setting the quantification ratio (shRNA-Bap31 transfected cells/control cells) of $>1.5$ as up-regulated threshold and $<0.67$ as down-regulated threshold $(p<0.05)$, there were 276 upregulated and 228 down-regulated proteins (Table 2 ). Then, we analyzed and counted the subcellular location, biological process, cellular component, and molecular function of Bap31-regulated proteins (Supplementary Tables S1-S4). The results indicate that Bap31 affected many important biological processes.

It is well recognized that Bap31 gene is located on the $\mathrm{X}$ chromosome, and its mutation causes $\mathrm{X}$-linked diseases 


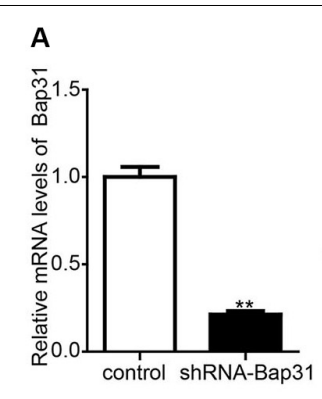

D
B

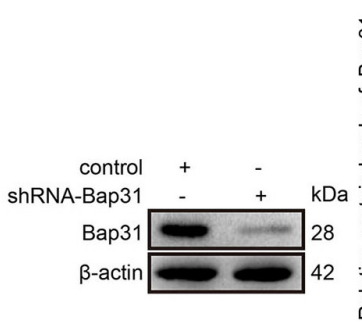

C

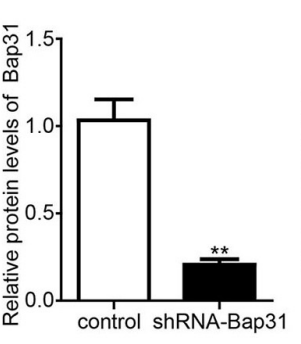

E

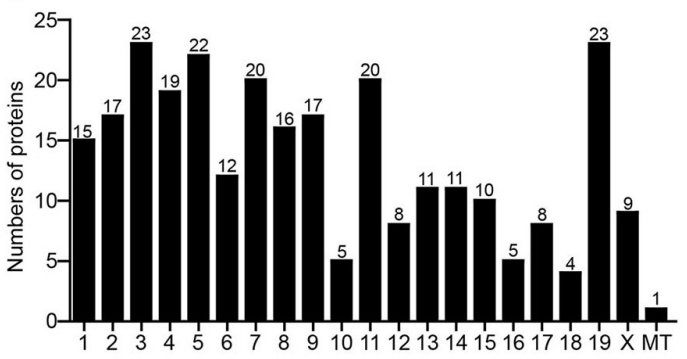

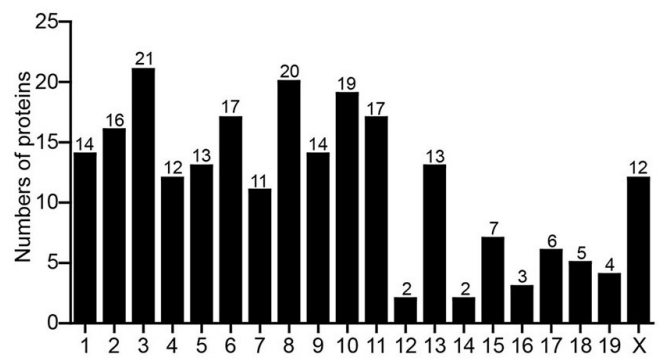

control
up-regulated proteins down-reguated proteins

FIGURE 1 | The located chromosome and X-linked Bap31 regulated proteins. Real-time PCR (A) and Western blot (B) analyses were used to detect the expression levels of Bap31 in shRNA-Bap31 transfected N2a cells. The isobaric tags for relative and absolute quantification technique was used to detect and analyze the different proteins between shRNA-Bap31 transfected cells and control cells. The numbers of up-regulated (C) and down-regulated (D) proteins were analyzed according to the differently located chromosomes in shRNA-Bap31 transfected cells. The genes regulated by Bap31 knockdown were located on the $X$ chromosome (E). Black: control, red: up-regulated genes, green: down-regulated genes. ${ }^{\star *} p<0.01$ vs. control groups, $n=3$.

(Osaka et al., 2012; Cacciagli et al., 2013). To explore the mechanism of Bap31 on X-linked diseases, we identified 21 $\mathrm{X}$-linked diseases-related proteins contained with 9 up-regulated (ABCB7, CCDC22, IGBP1, MAOA, MCTS1, MSN, PBDC1, PGK1, and SH3BGRL) and 12 down-regulated (HSD17B10, HUWE1, KIF4, MAGED1, PDHA1, PLXNA3, RBM3, SMS, TIMM17B, TSPAN7, UBA1, and VAP1) proteins from the iTRAQ results (Figures 1C-E and Supplementary Table S5). The findings support the interaction between Bap31 and X-linked diseases.

\section{Bap31 Negatively Regulates the Expression of MAOA}

Monoamine oxidase A is one of the Bap31-regulated proteins and up-regulated by the knockdown of Bap31 (Figure 1E). To further verify the results, Western blot analysis was used to detect the expression level of MAOA in shRNA-Bap31 transfected cells. The results showed that the protein level of MAOA was 3.14fold in shRNA-Bap31 transfected cells compared with that of the control cells (Figure 2A). Meanwhile, we also constructed Bap31

TABLE 2 | Summary of identified, quantified proteins and differentially quantified proteins $(>1.5$, or $<0.67)$.

\begin{tabular}{lcccc}
\hline Name & Identified & Quantified & $\begin{array}{c}\text { Up-regulated } \\
(>\mathbf{1 . 5})\end{array}$ & $\begin{array}{c}\text { Down- } \\
\text { regulated } \\
(<\mathbf{0 . 6 7 )}\end{array}$ \\
\hline Protein numbers & 4,349 & 2,989 & 276 & 228
\end{tabular}

overexpression cells transfected with Bap31-Flag plasmid and detected the expression level of MAOA. The results showed that the protein level of MAOA was $54.5 \%$ in Bap31 overexpression cells compared with that in control cells (Figure 2C). Besides that, we overexpressed Bap31-Flag in shRNA-Bap31 transfected cells, and Western blot analysis showed that the overexpressed Bap31 reversed the MAOA expression level which was increased by Bap31 knockdown (Figure 2E). Flow cytometry was used to further confirm that the levels of MAOA in Bap31 disturbed cells were significantly changed compared with that in the control cells; the same change trend as that of Western blot analysis was observed (Figures 2B,D). These results indicate that Bap31 negatively regulates the expression of MAOA.

\section{Bap31 Does Not Affect the Ubiquitination and Degradation Ratio of MAOA}

Monoamine oxidase A can be degraded by Rines E3 ubiquitin ligase (Kabayama et al., 2013), leading us to speculate that one possibility for the mechanism may be that Bap31 affected the ubiquitin-dependent proteasomal degradation of MAOA. To further explore this mechanism, we constructed the HEK293T cell line stably transfected with MAOA-HA plasmid (Supplementary Figure S1). After that, we transfected shRNABap31 to the cells and used an anti-HA antibody to pull down MAOA to exclude the effect of Bap31 on the regulation of endogenous MAOA mRNA levels. Western blot analysis of precipitated proteins with an anti-ub antibody showed that the ubiquitinated MAOA in shRNA-Bap31 transfected cells was not different from that in control cells (Figure 3A); the same 
A

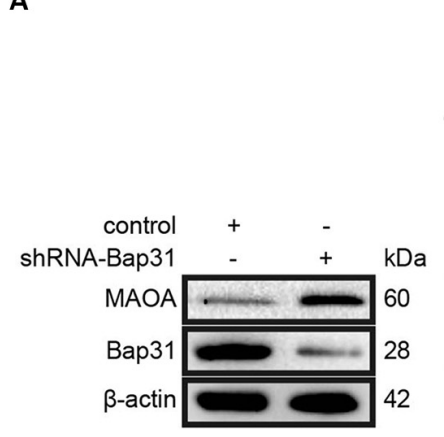

C

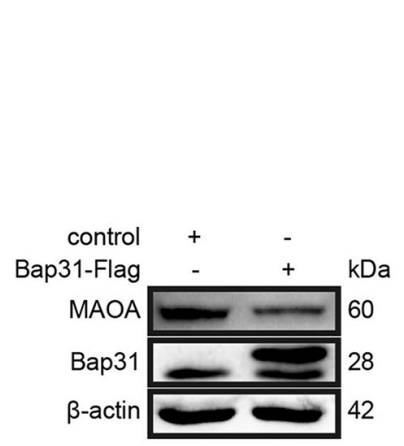

E

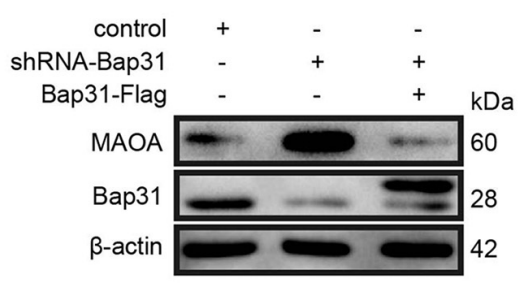

B
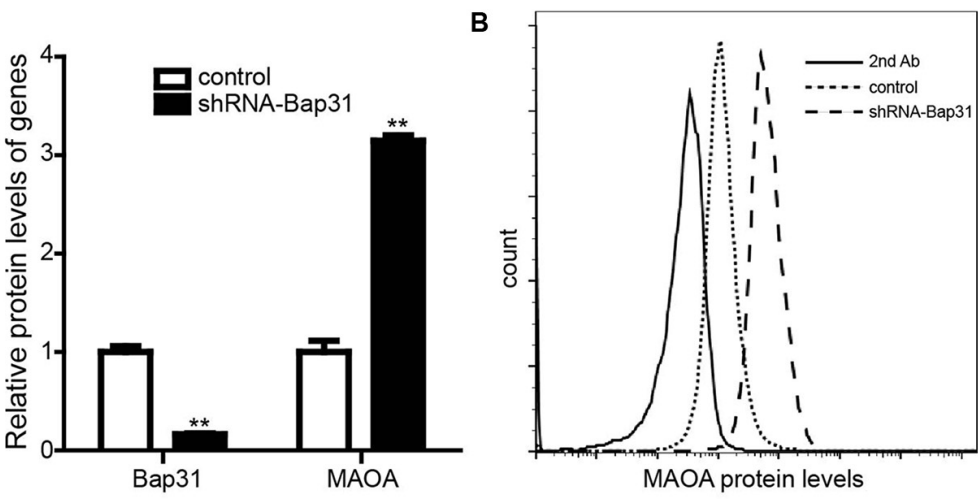

D

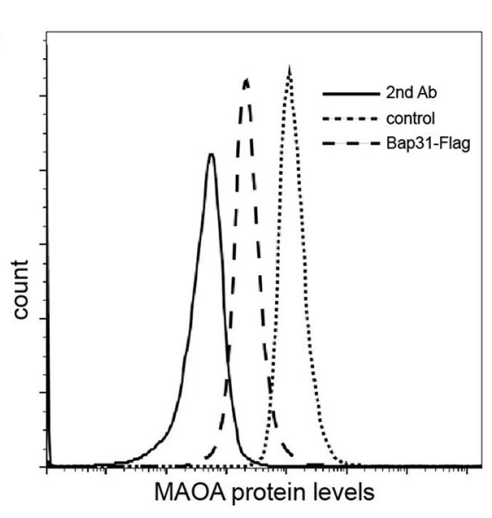

MAOA protein levels

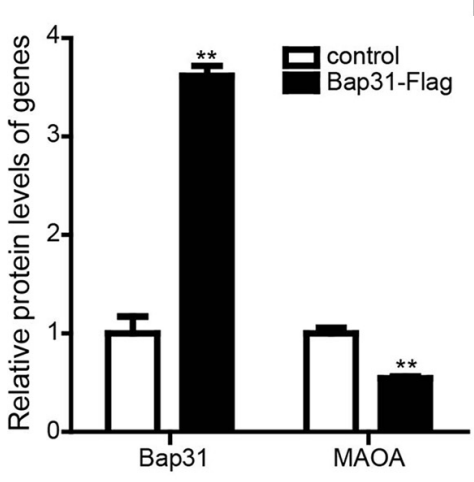

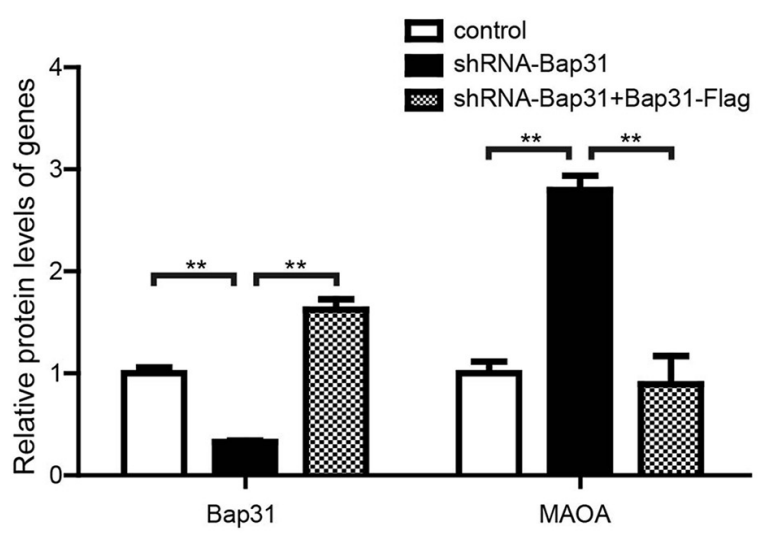

FIGURE 2 | The expression levels of monoamine oxidase A (MAOA) in shRNA-Bap31 and Bap31-Flag transfected cells. Western blot analyses were used to detect the protein levels of MAOA in shRNA-Bap31 (A) and Bap31-Flag (C) transfected cells. (B,D) Flow cytometry was used to detect the levels of MAOA in the same group as in panels $\mathbf{( A , C )}$. The protein levels of MAOA in shRNA-Bap31 transfected cells with overexpression of Bap31-Flag (E). The histogram shows the relative expression levels of targeted proteins in Bap31 disturbed groups compared with the control groups. ${ }^{* *} p<0.01$ vs. control groups, $n=3$.

results were obtained in Bap31 overexpressed cells (Figure 3B). The results demonstrated that Bap31 did not directly affect the ubiquitination of MAOA. Moreover, we detected the protein levels of MAOA and analyzed the degradation ratio in shRNABap31 transfected cells cultured with $10 \mu \mathrm{g} / \mathrm{ml}$ cycloheximide (CHX, one of the protein synthesis inhibitors) for $0,6,12,24$, 48, and $72 \mathrm{~h}$. The results showed that the degradation ratio of MAOA was not significantly changed by the knockdown of Bap31 (Figures 3C,E). Meanwhile, we detected the levels of MAOA in the Bap31 overexpressed cells. The results showed that the degradation ratios of MAOA either in the presence of Bap31-Flag or in the empty vector transfected cells were not significantly different (Figures 3D,F). Together, these results indicate that Bap31 does not directly affect the degradation of MAOA protein.

\section{Bap31 Regulates the mRNA Levels of MAOA via R1}

After ruling out a direct effect of Bap31 on the degradation of MAOA, we compared the amount of mRNA levels of MAOA in 
A

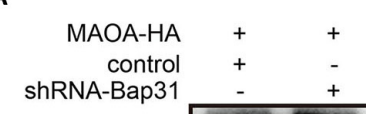

IP: HA

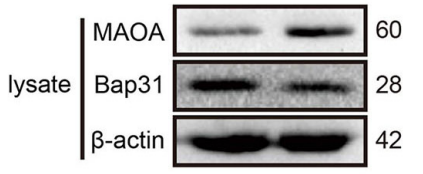

B
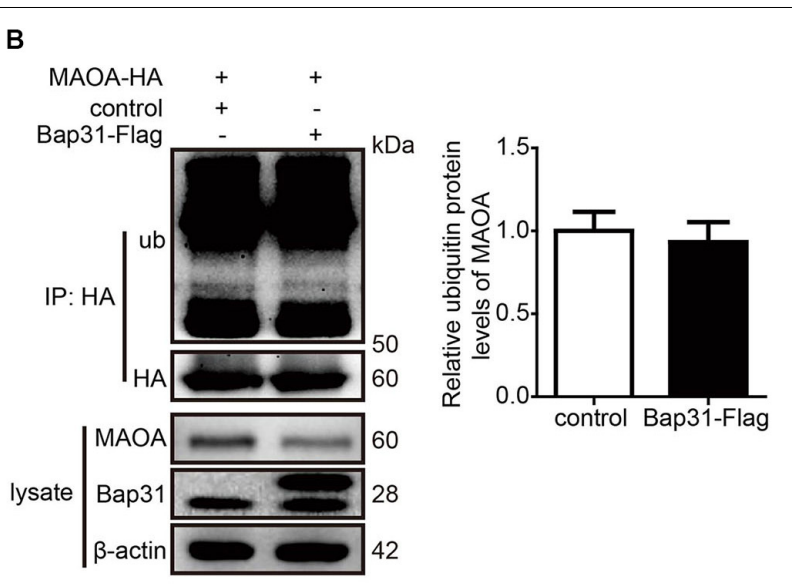

C

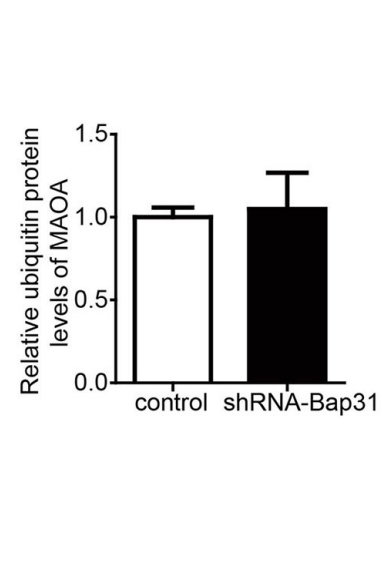

control

shRNA-Bap31

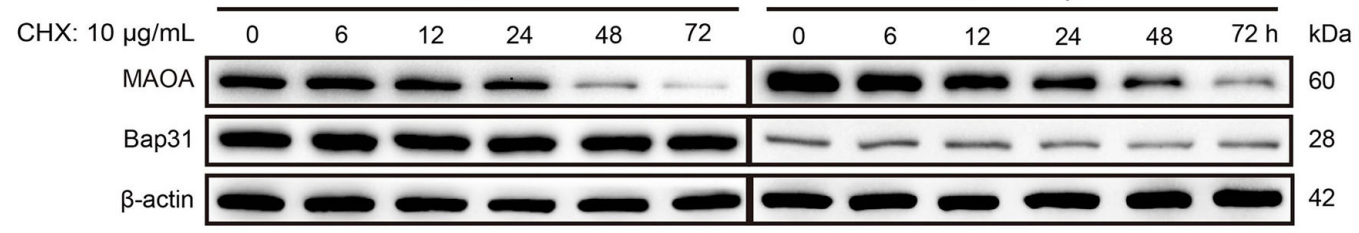

D

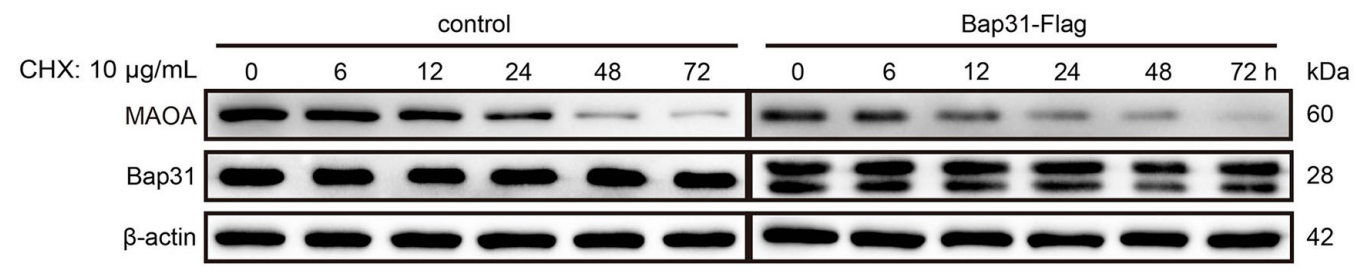

E
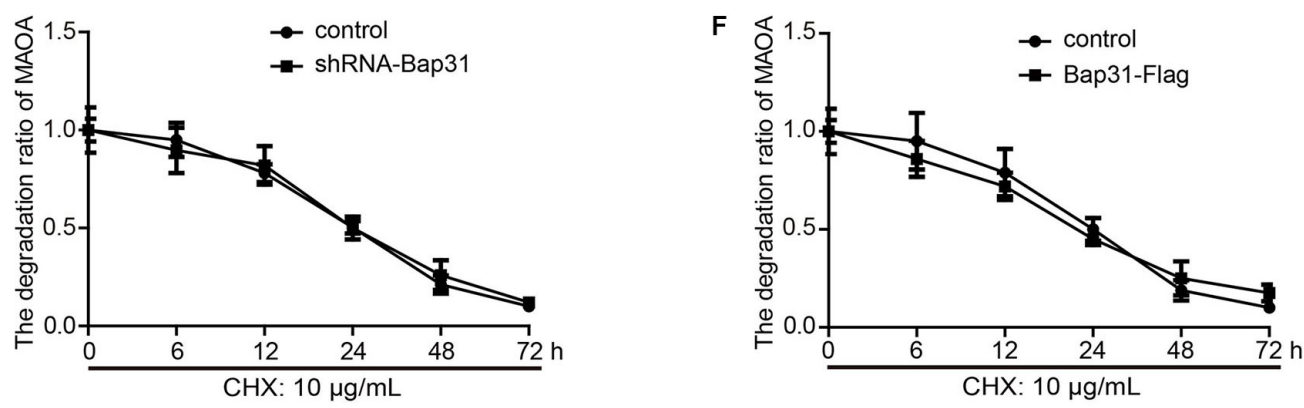

FIGURE 3 | Bap31 did not regulate the degradation of monoamine oxidase A (MAOA). MAOA-HA stable expression cells were transfected with shRNA-Bap31 (A) or Bap31-Flag (B) plasmids for $48 \mathrm{~h}$. The immunoprecipitated proteins were detected and showed the ubiquitinated MAOA levels. The histogram indicates the relative expression levels of ubiquitinated MAOA in Bap31 disturbed groups compared with the control groups. The same cells as in panels (A,B) were treated with $10 \mu \mathrm{g} / \mathrm{ml}$ $\mathrm{CHX}$ and then the protein levels of MAOA were determined at $0,6,12,24,48$, and $72 \mathrm{~h}$ (C,D). The line chart indicated the degradation ratio of MAOA in Bap31 disturbed groups and control groups cultured with $\mathrm{CHX}(\mathbf{E}, \mathbf{F}), n=3$.

shRNA-Bap31 transfected cells and control cells. Real-time PCR analyses showed that the mRNA level of MAOA in shRNA-Bap31 transfected cells was 2.53-fold compared with that of the control cells (Supplementary Figure S2).

SP1 (the transcription factor of MAOA) and R1 (the transcriptional repressor of MAOA) competitively bind with the MAOA promoter region and reversely regulate the expression of
MAOA (Ou et al., 2006a). To further explore the mechanism of Bap31 on the mRNA level of MAOA, we constructed the MAOA luciferase report plasmid containing the binding sites of SP1 and R1. Then, we transfected it into Bap31 disturbed cells. The results showed that the luciferase activities of MAOA were negatively regulated by Bap31 (Figures 4A,C). Western blot analyses showed that the protein level of R1 in shRNA-Bap31 transfected 


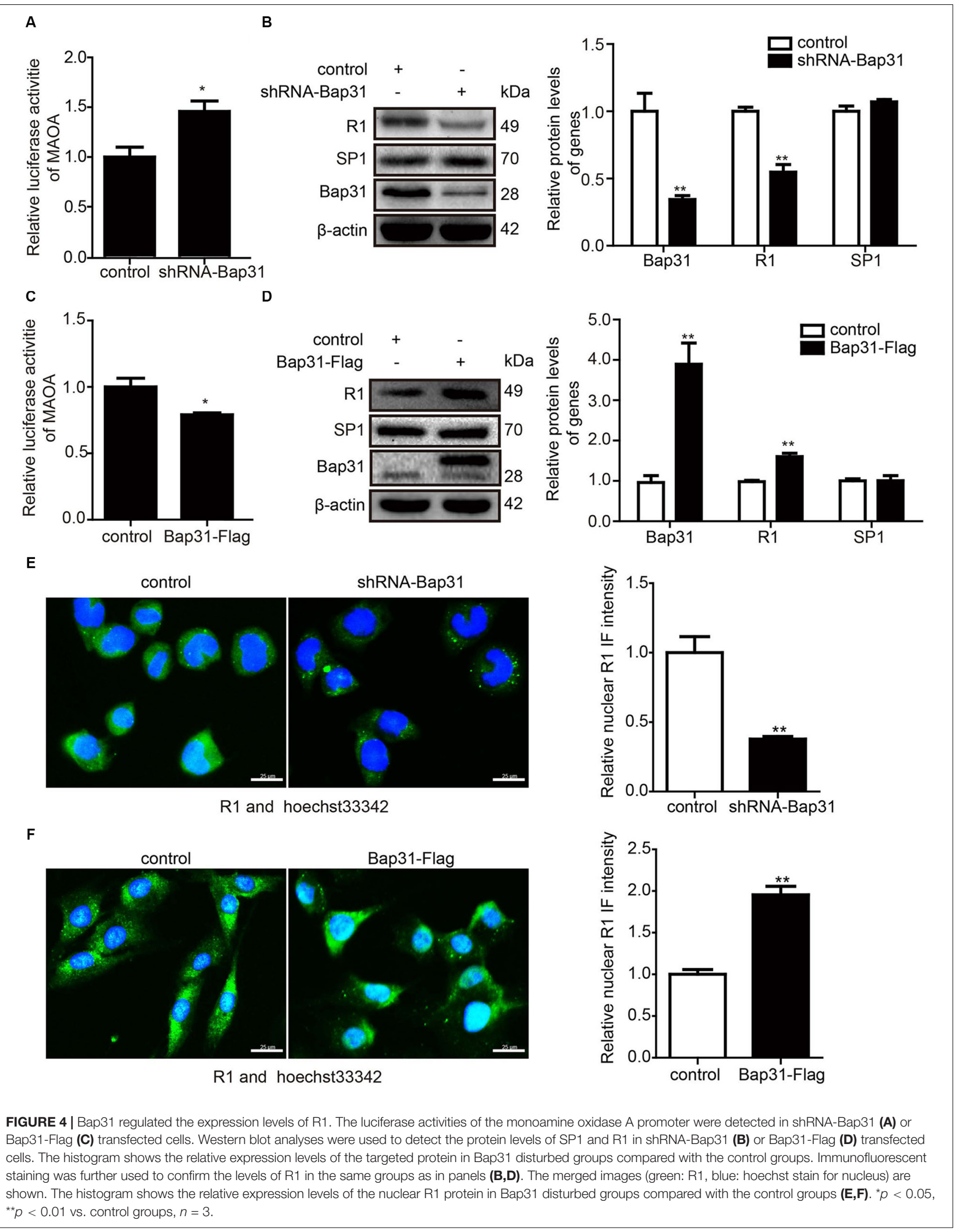



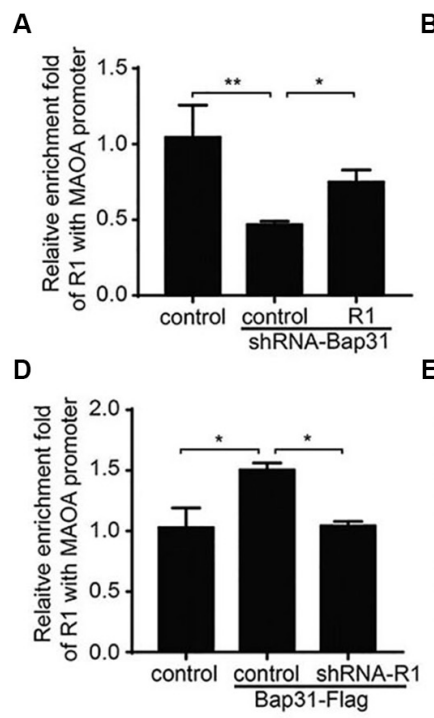

G

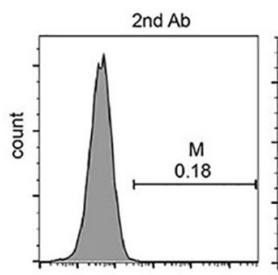

B
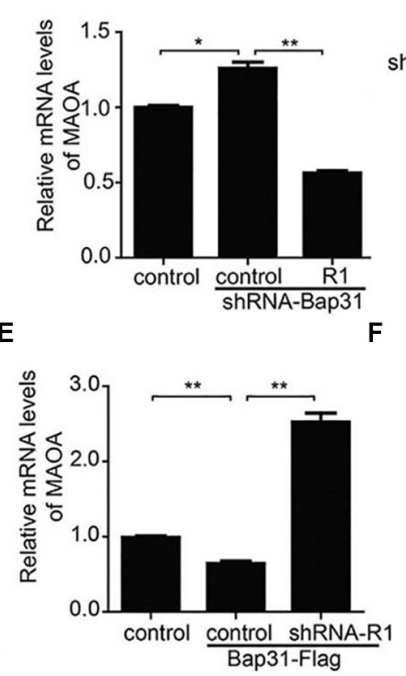

C
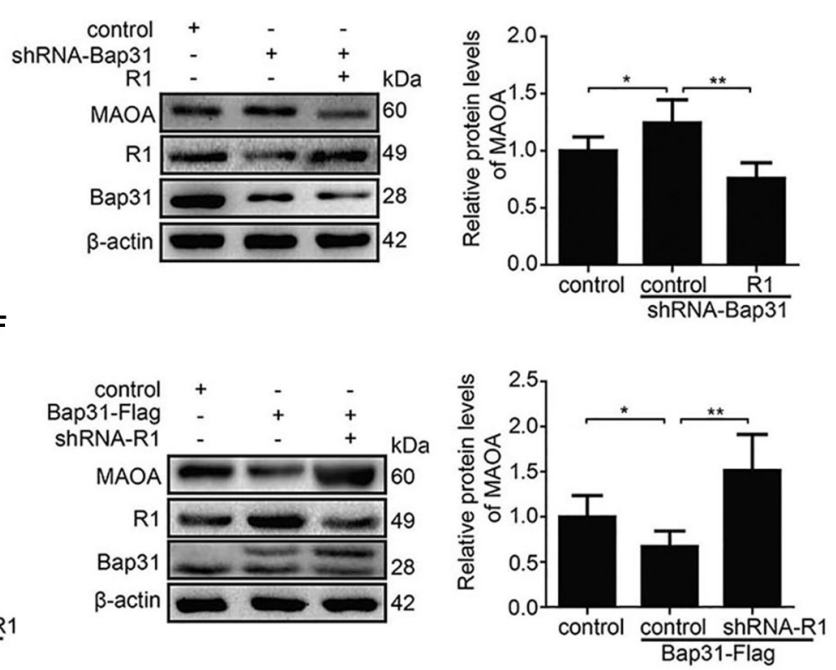

control
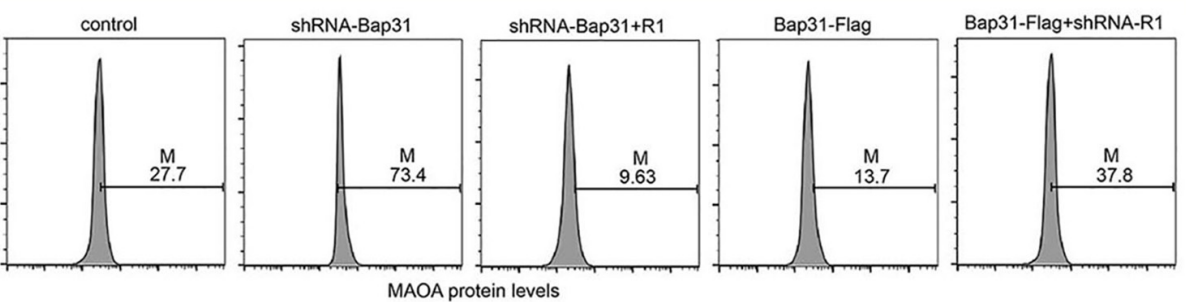

MAOA protein levels

FIGURE 5 | Bap31 regulated monoamine oxidase A (MAOA) expression through R1. ChIP was used to analyze the binding activities between R1 and MAOA promoter in Bap31 disturbed cells with R1 reverse interference (A,D). Real-time PCR and Western blot were used to detect the mRNA and the protein levels of MAOA in shRNA-Bap31 transfected cells with over-expressing R1 (B,C) or in Bap31-Flag transfected cells with knockdown of R1 (E,F). (G) Flow cytometry was used to detect the levels of MAOA in the same groups as in panels (A,D). The histogram shows the relative expression levels of the targeted protein. ${ }^{*} p<0.05$, ${ }^{\star *} p<0.01$ vs. control groups, $n=3$.

cells was $54.8 \%$ of that in the control cells (Figure 4B), and the protein level of R1 in Bap31-Flag overexpressed cells was 1.60fold of that in the control cells (Figure 4D). Immunofluorescent staining analysis obtained the same change trend as that of Western blot analysis (Figures $\mathbf{4 E}, \mathbf{F}$ ). However, the protein levels of SP1 did not have a significant difference among these groups (Figures 4B,D). These results imply that Bap31 regulates MAOA possibly via the regulation of $\mathrm{R} 1$.

To further explore the mechanism of Bap31 on MAOA via $\mathrm{R} 1$, we constructed R1 overexpression and shRNA-R1 plasmids and transfected them into Bap31 disturbed cells. Then, we used ChIP assay to explore whether Bap31 could impact MAOA gene transcription via R1. Our data revealed that the overexpression of $\mathrm{R} 1$ increased the binding activities of $\mathrm{R} 1$ to the MAOA promoter region which was reduced by the knockdown of Bap31 (Figure 5A). Conversely, the binding activities of $\mathrm{R} 1$ to the MAOA promoter region was increased by the overexpression of Bap31, and the knockdown of R1 in Bap31 over-expressed cells reduced the activities compared with the non-knockdown R1 groups (Figure 5D). Moreover, real-time PCR and Western blot were used to detect the levels of MAOA in Bap31 knockdown cells with overexpression of R1; the results showed that they were 44.7 and $60.9 \%$ compared with that of Bap31 knockdown cells, respectively (Figures 5B,C). Conversely, we inhibited R1 by transfection shRNA-R1 plasmid into Bap31 over-expressed cells. The mRNA and protein levels of MAOA in these cells were 3.93- and 2.26-fold compared to the Bap31 over-expressed cells (Figures 5E,F). Furthermore, the results of flow cytometry showed the same change trend as that of real-time PCR and Western blot analyses (Figure 5G). These results demonstrate that Bap31 regulates the levels of MAOA via R1.

\section{DISCUSSION AND CONCLUSION}

Bap31 is highly expressed in the ER and its gene is located on the X chromosome (Adachi et al., 1996). It participates in transporting member proteins from the ER to other organelles, mediating apoptosis via p20 and regulating the ERAD pathway (Breckenridge et al., 2003; Wakana et al., 2008). Our recent study indicates that Bap31 deficiency leads to the formation of amyloid- $\beta$ plaques via reducing RTN3 stability in Alzheimer's disease (Wang et al., 2019). The conditional knockout of BAP31 in hepatocyte promotes SREBP1C activation and hepatic lipid accumulation and worsens insulin resistance in HFD-induced obesity in mice (Xu et al., 2018). Bap31 regulates the expression 
of VCP via its transcription factor Elf2 (Jia et al., 2018). However, there is still little research on the biological function of Bap31. ITRAQ-based proteomic technique as a new technique is used to detect the whole proteomics among different groups. In this study, iTRAQ was used to detect and analyze the proteins regulated by Bap31. In analyzing the results, there are 504 differentially expressed proteins in Bap31 knockdown cells: 276 up-regulated and 228 down-regulated proteins. These proteins participated in many important biological process and cellular component and molecular function, for example, biological regulation, metabolic process, cellular component organization or biogenesis, multicellular organismal process, developmental process, immune system process, and so on (Supplementary Tables S1-S5). The results of iTRAQ provided the basis for studying the unknown function of Bap31.

The contiguous deletion of SLC6A8 and Bap31 is related to hearing loss and liver dysfunction (Osaka et al., 2012). Bap31 mutations cause a severe X-linked phenotype with deafness, dystonia, and central hypomyelination (Cacciagli et al., 2013). Besides that, the contiguous ABCD1 DXS1357E (Bap31) deletion syndrome (CADDS) is $90 \mathrm{~kb}$, ranging from exon 4 of Bap31 to exon 8 of PDZD4 and contained ABCD1, PLXNB3, SRPK3, IDH3G, and SSR4 (Corzo et al., 2002). Based on these, we speculated that Bap31 may be an important factor in mediating $\mathrm{X}$-linked diseases. In this study, the analysis of the 504 differentially expressed proteins regulated by Bap31 showed that there were 21 genes located on the $\mathrm{X}$ chromosome containing 9 up-regulated and 12 down-regulated proteins (Figure 1). Our results further demonstrated the function of Bap31 on X-linked diseases.

Monoamine oxidase $\mathrm{A}$ is a flavoenzyme located on the outer mitochondrial membrane. It can catalyze the oxidative deamination of serotonin, dopamine, and norepinephrine (Yu et al., 2014). Mutations of MAOA cause X-linked diseases (Chen et al., 1993) and increased risk for Parkinson's disease (Hotamisligil et al., 1994) and Norrie disease (Wolff et al., 1992). Moreover, MAOA participates in apoptotic signaling pathways (Ou et al., 2006b) and Shh-IL6-Rankl signaling pathway (Wu et al., 2017). However, whether Bap31 regulates the expression of MAOA was unknown. Our results showed that Bap31 negatively regulates the expression of MAOA, whether knockdown or overexpression of Bap31 (Figure 2). To explore the mechanism of Bap31 on MAOA, we firstly detected the regulation of Bap31 on the degradation of MAOA. Although Bap31 affects the degradation of many proteins (Wang et al., 2008; Chen et al., 2019), the regulation of Bap31 on MAOA expressions does not appear to be dependent on these effects. Bap31 did not directly affect the ubiqutin-mediated degradation of MAOA in MAOA-HA stable cells with knockdown or overexpression of Bap31 (Figures 3A,B). Consistent with this, Bap31 hardly changed the degradation ratio of MAOA compared to its controls (Figures 3C-F). It suggests that Bap31 does not exert its direct effect on the MAOA protein degradation level. Rather intriguingly, the depletion of Bap31 with shRNA-Bap31 strongly increased the amounts of mRNA for MAOA (Supplementary Figure S2). Furthermore, Bap31 did not affect the ubiquitinmediated degradation level of transfected MAOA-HA protein expression driven by a CMV promoter in the pcDNA plasmid (Figures 3A,B). These led us to further verify that Bap31 mediates the transcription of MAOA. R1, the transcriptional repressor of MAOA, was down-regulated and caused apoptosis in serum starvation (Ou et al., 2006b). Consistent with the former study, our data showed that knockdown or overexpression of $\mathrm{R} 1$ affected the binding activities of $\mathrm{R} 1$ with the MAOA promoter, thereby negatively regulating the mRNA and protein levels of MAOA. Besides that, our results also indicated that Bap31 regulates the expression levels of R1 (Figure 4). Ou et al. (2006b) have found that Bcl-2 was decreased and caused the down-regulation of the level of R1 in serum starvationinduced apoptosis. Previous studies indicate that Bap31 is identified as a Bcl-2 interacting protein ( $\mathrm{Ng}$ et al., 1997) and the cleavage of Bap31 (p20) as the upstream of Bcl-2 affects cell surface properties (Stojanovic et al., 2005). One of the possible mechanisms of Bap31 on R1 may be via Bcl-2. However, the molecular mechanism of Bap31 on R1 still needs further exploration.

In summary, our findings indicate that Bap31 regulates MAOA possibly via its effect on $\mathrm{R} 1$ and support the functions of Bap31 in X-linked diseases. Our work helps to uncover the molecular mechanism of Bap31 on X-linked diseases and may help find the potential molecular targets for X-linked disease therapy.

\section{DATA AVAILABILITY STATEMENT}

The raw data supporting the conclusions of this manuscript will be made available by the authors, without undue reservation, to any qualified researcher.

\section{AUTHOR CONTRIBUTIONS}

CJ wrote the manuscript and researched the data. GL researched and analyzed the data. RJ helped with revising the discussion. XL and QY helped with analyzing the data. WL helped with revising the manuscript. $\mathrm{YH}$ directed the study and revised the manuscript. BW directed the study, analyzed and approved all of the data, and edited the manuscript.

\section{FUNDING}

This work was funded by the LiaoNing Revitalization Talents Program (XLYC1902063), the National Natural Science Foundation of China (31670770, 31370784, 2016YFC1302402, U1603125, and 81473330), and the Fundamental Research Funds for the Central Universities of China (N182008004 and N182006001).

\section{SUPPLEMENTARY MATERIAL}

The Supplementary Material for this article can be found online at: https://www.frontiersin.org/articles/10.3389/fmolb.2020. 00064/full\#supplementary-material 


\section{REFERENCES}

Abe, F., Van Prooyen, N., Ladasky, J. J., and Edidin, M. (2009). Interaction of Bap31 and MHC class I molecules and their traffic out of the endoplasmic reticulum. J. Immunol. 182, 4776-4783. doi: 10.4049/jimmunol.0800242

Adachi, T., Schamel, W. W., Kim, K. M., Watanabe, T., Becker, B., Nielsen, P. J., et al. (1996). The specificity of association of the IgD molecule with the accessory proteins BAP31/BAP29 lies in the IgD transmembrane sequence. EMBO J. 15, 1534-1541. doi: 10.1002/j.1460-2075.1996.tb00497.x

Annaert, W. G., Becker, B., Kistner, U., Reth, M., and Jahn, R. (1997). Export of cellubrevin from the endoplasmic reticulum is controlled by BAP31. J. Cell. Biol. 139, 1397-1410. doi: 10.1083/jcb.139.6.1397

Breckenridge, D. G., Stojanovic, M., Marcellus, R. C., and Shore, G. C. (2003). Caspase cleavage product of BAP31 induces mitochondrial fission through endoplasmic reticulum calcium signals, enhancing cytochrome $c$ release to the cytosol. J. Cell. Biol. 160, 1115-1127. doi: 10.1083/jcb.200212059

Cacciagli, P., Sutera-Sardo, J., Borges-Correia, A., Roux, J. C., Dorboz, I., Desvignes, J. P., et al. (2013). Mutations in BCAP31 cause a severe $\mathrm{X}$-linked phenotype with deafness, dystonia, and central hypomyelination and disorganize the Golgi apparatus. Am. J. Hum. Genet. 93, 579-586. doi: 10.1016/ j.ajhg.2013.07.023

Chen, J., Guo, H., Jiang, H., Namusamba, M., Wang, C., Lan, T., et al. (2019). A BAP31 intrabody induces gastric cancer cell death by inhibiting p27 (kip1) proteasome degradation. Int. J. Cancer 144, 2051-2062. doi: 10.1002/ijc.31930

Chen, Z. Y., Battinelli, E. M., Fielder, A., Bundey, S., Sims, K., Breakefield, X. O., et al. (1993). A mutation in the Norrie disease gene (NDP) associated with X-linked familial exudative vitreoretinopathy. Nat. Genet. 5, 180-183. doi: 10.1038/ng1093-180

Chen, Z. Y., Denney, R. M., and Breakefield, X. O. (1995). Norrie disease and MAO genes: nearest neighbors. Hum. Mol. Genet. 4, 1729-1737. doi: 10.1093/hmg/4. suppl_1.1729

Corzo, D., Gibson, W., Johnson, K., Mitchell, G., LePage, G., Cox, G. F., et al. (2002). Contiguous deletion of the $\mathrm{X}$-linked adrenoleukodystrophy gene $(A B C D 1)$ and DXS1357E: a novel neonatal phenotype similar to peroxisomal biogenesis disorders. Am. J. Hum. Genet. 70, 1520-1531. doi: 10.1086/340849

Gupta, M., Neavin, D., Liu, D., Biernacka, J., Hall-Flavin, D., Bobo, W. V., et al. (2016). TSPAN5, ERICH3 and selective serotonin reuptake inhibitors in major depressive disorder: pharmacometabolomics-informed pharmacogenomics. Mol. Psychiatry 21, 1717-1725. doi: 10.1038/mp.2016.6

Hotamisligil, G. S., Girmen, A. S., Fink, J. S., Tivol, E., Shalish, C., Trofatter, J., et al. (1994). Hereditary variations in monoamine-oxidase as a risk factor for Parkinson's disease. Mov. Disord. 9, 305-310. doi: 10.1002/mds.870090304

Iwasa, M., Yamagata, T., Mizuguchi, M., Itoh, M., Matsumoto, A., Hironaka, M., et al. (2013). Contiguous ABCD1 DXS1357E deletion syndrome: report of an autopsy case. Neuropathology 33, 292-298. doi: 10.1111/j.1440-1789.2012. 01348.x

Jia, C. C., Du, J., Liu, X., Jiang, R., Huang, Y., Wang, T., et al. (2018). B-cell receptorassociated protein 31 regulates the expression of valosin-containing protein through Elf2. Cell. Physiol. Biochem. 51, 1799-1814. doi: 10.1159/000495682

Kabayama, M., Sakoori, K., Ornthanalai, V. G., and Aruga, J. (2013). Rines E3 ubiquitin ligase regulates MAO-A levels and emotional responses. J. Neurosci. 33, 12940-12953. doi: 10.1523/JNEUROSCI.5717-12.2013

Määttä, J., Hallikas, O., Welti, S., Hildén, P., Schröder, J., and Kuismanen, E. (2000). Limited caspase cleavage of human BAP31. FEBS Lett. 484, 202-206. doi: 10.1016/s0014-5793(00)02159- 1

Manca, M., Pessoa, V., Lopez, A. I., Harrison, P. T., Miyajima, F., Sharp, H., et al. (2018). The regulation of monoamine oxidase a gene expression by distinct variable number tandem repeats. J. Mol. Neurosci. 64, 459-470. doi: 10.1007/ s12031-018-1044-z

Manley, H. A., and Lennon, V. A. (2001). Endoplasmic reticulum membranesorting protein of lymphocytes (BAP31) is highly expressed in neurons and discrete endocrine cells. J. Histochem. Cytochem. 49, 1235-1243. doi: 10.1177/ 002215540104901005

Moulder, R., Bhosale, S. D., Goodlett, D. R., and Lahesmaa, R. (2018). Analysis of the plasma proteome using iTRAQ and TMT-based lsobaric labeling. Mass. Spectrom. Rev. 37, 583-606. doi: 10.1002/mas. 21550
Ng, F. W. H., Nguyen, M., Kwan, T., Branton, P. E., Nicholson, D. W., Cromlish, J. A., et al. (1997), p28 Bap31, a Bcl-2/Bcl$\mathrm{XL}-$ and procaspase-8-associated protein in the endoplasmic reticulum. J. Cell. Biol. 139, 327-338. doi: 10.1083/jcb.139. 2.327

Niu, K., Xu, J., Cao, Y., Hou, Y., Shan, M., Wang, Y., et al. (2017). BAP31 is involved in T cell activation through TCR signal pathways. Sci. Rep. 7:44809. doi: 10.1038/srep44809

Osaka, H., Takagi, A., Tsuyusaki, Y., Wada, T., Iai, M., Yamashita, S., et al. (2012). Contiguous deletion of SLC6A8 and BAP31 in a patient with severe dystonia and sensorineural deafness. Mol. Genet. Metab. 106, 43-47. doi: 10.1016/j. ymgme.2012.02.018

Ou, X.-M., Chen, K., and Shih, J. C. (2006a). Glucocorticoid and androgen activation of monoamine oxidase $\mathrm{A}$ is regulated differently by $\mathrm{R} 1$ and Sp1. J. Biol. Chem. 281, 21512-21525. doi: 10.1074/jbc.M60025 0200

Ou, X.-M., Chen, K., and Shih, J. C. (2006b). Monoamine oxidase A and repressor $\mathrm{R} 1$ are involved in apoptotic signaling pathway. Proc. Natl. Acad. Sci. U.S.A. 103, 10923-10928. doi: 10.1073/pnas.0601515103

Schluter, T., Winz, O., Henkel, K., Eggermann, T., Mohammadkhani-Shali, S., Dietrich, C., et al. (2016). MAOA-VNTR polymorphism modulates contextdependent dopamine release and aggressive behavior in males. Neuroimage 125, 378-385. doi: 10.1016/j.neuroimage.2015.10.031

Son, S. Y., Ma, J., Kondou, Y., Yoshimura, M., Yamashita, E., and Tsukihara, T. (2008). Structure of human monoamine oxidase A at 2.2-A resolution: the control of opening the entry for substrates/inhibitors. Proc. Natl. Acad. Sci. U.S.A. 105, 5739-5744. doi: 10.1073/pnas.0710626105

Stojanovic, M., Germain, M., Nguyen, M., and Shore, G. C. (2005). BAP31 and its caspase cleavage product regulate cell surface expression of tetraspanins and integrin-mediated cell survival. J. Biol. Chem. 280, 30018-30024. doi: 10.1074/jbc.M501306200

Verma, D., Chakraborti, B., Karmakar, A., Bandyopadhyay, T., Singh, A. S., Sinha, S., et al. (2014). Sexual dimorphic effect in the genetic association of monoamine oxidase A (MAOA) markers with autism spectrum disorder. Prog. Neuropsychopharmacol. Biol. Psychiatry 50, 11-20. doi: 10.1016/j.pnpbp.2013. 11.010

Wakana, Y., Takai, S., Nakajima, K., Tani, K., Yamamoto, A., Watson, P., et al. (2008). Bap31 is an itinerant protein that moves between the peripheral endoplasmic reticulum (ER) and a juxtanuclear compartment related to ERassociated Degradation. Mol. Biol. Cell. 19, 1825-1836. doi: 10.1091/mbc.E0708-0781

Wang, B., Heath-Engel, H., Zhang, D., Nguyen, N., Thomas, D. Y., Hanrahan, J. W., et al. (2008). BAP31 interacts with Sec61 translocons and promotes retrotranslocation of CFTRDeltaF508 via the derlin-1 complex. Cell 133, 10801092. doi: 10.1016/j.cell.2008.04.042

Wang, B., Pelletier, J., Massaad, M. J., Herscovics, A., and Shore, G. C. (2004). The yeast split-ubiquitin membrane protein two-hybrid screen identifies BAP31 as a regulator of the turnover of endoplasmic reticulum-associated protein tyrosine phosphatase-like B. Mol. Cell. Biol. 24, 2767-2778. doi: 10.1128/mcb.24.7.27672778.2004

Wang, T., Chen, J., Hou, Y., Yu, Y., and Wang, B. (2019). BAP31 deficiency contributes to the formation of amyloid- $\beta$ plaques in Alzheimer's disease by reducing the stability of RTN3. FASEB. J. 33, 4936-4946. doi: 10.1096/fj. 201801702R

Wiese, S., Reidegeld, K. A., Meyer, H. E., and Warscheid, B. (2007). Protein labeling by iTRAQ: a new tool for quantitative mass spectrometry in proteome research. Proteomics 7, 340-350. doi: 10.1002/pmic.200600422

Wilson, J. D., and Barlowe, C. (2010). Yetlp and Yet3p, the yeast homologs of BAP29 and BAP31, interact with the endoplasmic reticulum translocation apparatus and are required for inositol prototrophy. J. Biol. Chem. 285, 18252 18261. doi: 10.1074/jbc.M109.080382

Wolff, G., Mayerová, A., Wienker, T. F., Atalianis, P., Ioannou, P., and Warburg, M. (1992). Clinical reinvestigation and linkage analysis in the family with Episkopi blindness (Norrie disease). J. Med. Genet. 29, 816-819. doi: 10.1136/jmg.29. 11.816

Wu, J. B., Yin, L., Shi, C., Li, Q., Duan, P., Huang, J. M., et al. (2017). MAOAdependent activation of Shh-IL6-RANKL signaling network promotes prostate 
cancer metastasis by engaging tumor-stromal cell interactions. Cancer Cell. 31, 368-382. doi: 10.1016/j.ccell.2017.02.003

Xu, J. L., Li, L. Y., Wang, Y. Q., Li, Y. Q., Shan, M., Sun, S. Z., et al. (2018). Hepatocyte-specific deletion of BAP31 promotes SREBP1C activation, promotes hepatic lipid accumulation, and worsens IR in mice. J. Lipid Res. 59, 35-47. doi: 10.1194/jlr.M077016

Yu, Q., Teixeira, C. M., Mahadevia, D., Huang, Y., Balsam, D., Mann, J. J., et al. (2014). Dopamine and serotonin signaling during two sensitive developmental periods differentially impact adult aggressive and affective behaviors in mice. Mol. Psychiatry 19, 688-698. doi: 10.1038/mp. 2014.10

Zen, K., Utech, M., Liu, Y., Soto, I., Nusrat, A., and Parkos, C. A. (2004). Association of BAP31 with CD11b/CD18. Potential role in intracellular trafficking of CD11b/CD18 in neutrophils. J. Biol. Chem. 279, 44924-44930. doi: 10.1074/jbc.M402115200
Zhang, Y., Ming, Q. S., Yi, J. Y., Wang, X., Chai, Q. L., and Yao, S. Q. (2017). Genegene-environment interactions of serotonin transporter, monoamine oxidase $\mathrm{A}$ and childhood maltreatment predict aggressive behavior in chinese adolescents. Front. Behav. Neurosci. 11:17. doi: 10.3389/fnbeh.2017.00017

Conflict of Interest: The authors declare that the research was conducted in the absence of any commercial or financial relationships that could be construed as a potential conflict of interest.

Copyright (C) 2020 Jia, Li, Jiang, Liu, Yuan, Le, Hou and Wang. This is an open-access article distributed under the terms of the Creative Commons Attribution License (CC BY). The use, distribution or reproduction in other forums is permitted, provided the original author(s) and the copyright owner(s) are credited and that the original publication in this journal is cited, in accordance with accepted academic practice. No use, distribution or reproduction is permitted which does not comply with these terms. 